\title{
Respuesta de la Sociedad Mexicana de Medicina Física y Rehabilitación ante la pandemia COVID-19
}

\section{Response of the Mexican Society of Physical Medicine and Rehabilitation to the COVID-19 pandemic}

Dr. Ángel Óscar Sánchez Ortiz

T a aparición de la COVID ha enfrentado a $\_$la humanidad a retos que pocos hubiéramos podido imaginar. Estos desafíos marcarán nuestro futuro en forma indeleble, así como la capacidad de adaptación ante las adversidades. Las respuestas ante estos reveses debían encontrarse en forma rápida y efectiva a fin de no trastocar fuertemente la marcha de nuestra civilización. Es por esto que la Sociedad Mexicana de Medicina Física y Rehabilitación, durante el bienio 2019-2020, presentó innovadoras respuestas ante este nuevo escenario, marcado por la pandemia del coronavirus.

Podemos comentar que durante el año 2019 y hasta marzo del 2020, cuando se presentó la emergencia en México, se llevaron a cabo las actividades en forma tradicional y presencial, tales como: sesiones mensuales, talleres y jornadas médicas de ingreso de nuevos socios. A partir del mes de abril del año 2020, una vez que se presentó la suspensión de actividades y el encierro masivo, se plantearon opciones de tipo tecnológico para poder cumplir los objetivos programados. A partir del mes de junio del año 2020, se reiniciaron las actividades científicas y de difusión habitual de la Sociedad, principalmente las sesiones mensuales, así como el XXVI Congreso Mexicano de Medicina Física y Rehabilitación y Tercer Congreso Internacional «Actualización en Rehabilitación». Mediante el uso de la alta tecnología disponible como el uso de Zoom, redes sociales y plataformas propias, pudimos llevar a cabo todas la actividades programadas y tradicionales de la Sociedad. También pudimos notar con agrado que incluso se logró una mayor difusión de todas nuestras sesiones, ya que muchos países, principalmente de Latinoamérica, América del Norte y España, se unieron entusiastamente a todas nuestras actividades. Con ello obtuvimos una mayor audiencia a todos nuestros eventos.

EI XXVI Congreso Mexicano de Medicina Física y Rehabilitación y Tercer Congreso Internacional «Actualización en Rehabilitación», programados en formato presencial para octubre 2020, se adaptaron para llevarse a cabo en enero del 2021 en formato virtual. Contó con una gran asistencia y sentó el precedente, en nuestra especialidad, como el primer Congreso Virtual de Rehabilitación en Latinoamérica y uno de los primeros Congresos Virtuales de Rehabilitación a nivel mundial. Esto permitió el intercambio de conocimientos médicos que se fueron adquiriendo durante los meses previos de la pandemia en México y a nivel internacional, lo que brindó herramientas para el tratamiento rehabilitatorio de los pacientes postCOVID-19 y del resto de pacientes con alguna discapacidad, en forma oportuna y eficiente.
* Presidente de la Sociedad Mexicana de Medicina Física y Rehabilitación. Bienio 2019-2020.
Citar como: Sánchez OÁÓ. Respuesta de la Sociedad Mexicana de Medicina Física y Rehabilitación ante la pandemia COVID-19. Rev Mex Med Fis Rehab. 2020; 32 (3-4): 36-37. https://dx.doi.org/10.35366/101968 
Fue particularmente notoria la rápida respuesta a la capacitación en la rehabilitación de todos los pacientes que sufrieron la COVID. Desde el mes de junio, iniciamos la difusión de las medidas de prevención y tratamiento del síndrome post-COVID, así como de las experiencias que nuestro país había tenido en la pandemia de la influenza H1N1 en el año 2009. Estos temas se convirtieron en preponderantes durante las sesiones científicas, congresos y en la revista de nuestra Sociedad.

El ingreso de nuevos socios, a través de las Jornadas para Médicos Residentes, se realizó también, por primera ocasión, en formato virtual.

La revista de la Sociedad Mexicana de Medicina Física y Rehabilitación se convierte en el Órgano de Difusión Científica de Rehabilitación de Iberoamérica. Ello obligó a buscar nuevas opciones de trabajo para el Comité Editorial que apoyen para la revisión más rápida y ordenada de los artículos que se envían para publicación a través de la revista. A finales del año 2020, se estableció una plataforma para este fin. Sin embargo, por la suspensión de actividades no esenciales, se ve interrumpida la impresión y distribución de la revista, en el tiempo de mayor contagio, en nuestro país, reestableciéndose posteriormente.

Nos tuvimos que capacitar mediante cursos intensivos en recursos tecnológicos digitales, para contar con opciones de aprendizaje y divulgación de la información médica científica. Por ello, durante el bienio 2019-2020, se plantearon nuevos paradigmas en la educación médica y la enseñanza continua en rehabilitación y, con ello, fortalezas en nuestro quehacer como Sociedad, en beneficio de nuestros pacientes y nuestros compañeros médicos.

La disposición hacia el cambio, tanto de los socios como de los miembros de la mesa directiva, permitió el logro de los objetivos y nuestra Sociedad resultó fortalecida y preparada para nuevos retos y circunstancias.

Sigamos adelante por el futuro de la Medicina Física y Rehabilitación, en beneficio de los pacientes y de la sociedad en general.

$$
\begin{aligned}
& \text { Correspondencia: } \\
& \text { Dr. Ángel Óscar Sánchez Ortiz } \\
& \text { E-mail: aoso4712@gmail.com }
\end{aligned}
$$

